\title{
Isoaspartylation Appears to Trigger Small Cell Lung Cancer-Associated Autoimmunity against
}

\section{Neuronal Protein ELAVL4}

Mario A. Pulido ${ }^{1 *}$, Meleeneh Kazarian DerHartunian ${ }^{1 *}$, Zhenxia Qin $^{2}$, Eric M. Chung ${ }^{1}$, Diane S. Kang ${ }^{1}$, Andrew W. Woodham ${ }^{3}$, Jeffrey A. Tsou ${ }^{1}$, Rinse Klooster, Omid Akbari ${ }^{3}$, Lina Wang ${ }^{4}$, W. Martin Kast ${ }^{3}$, Stephen V. Liu ${ }^{5}$, Jan J.G.M. Verschuuren ${ }^{6}$, Dana W. Aswad ${ }^{2}$, and Ite A. Laird-Offringa ${ }^{1}$.

*Mr. Pulido and Dr. DerHartunian contributed equally to this article.

Authors' Affiliations: ${ }^{1}$ Departments of Surgery and of Biochemistry and Molecular Medicine, Norris Comprehensive Cancer Center, Keck School of Medicine, University of Southern California, Los Angeles, CA; ${ }^{2}$ Department of Molecular Biology and Biochemistry, University of California at Irvine, Irvine, CA; ${ }^{3}$ Department of Molecular Microbiology and Immunology Norris Comprehensive Cancer

Center, Keck School of Medicine, University of Southern California, Los Angeles, CA; ${ }^{4}$ Department of Pathology, Keck School of Medicine, University of Southern California, Los Angeles, CA; ${ }^{5}$ Department of Medicine, Norris Comprehensive Cancer Center, Keck School of Medicine, University of Southern California, Los Angeles, CA; ${ }^{6}$ Department of Neurology, Leiden University, Leiden, the Netherlands. Note: Supplementary data for this article are available on line.

Running head: Isoaspartylation in SCLC autoimmunity

Corresponding Author: Ite A. Laird-Offringa, USC/Norris Comprehensive Cancer Center, 1441

Eastlake Ave. NTT6420, Los Angeles, CA 90089-9176, USA; Phone: (323) 865-0655; Fax: (323) 865-

0158; E-mail: $\underline{\text { ilaird@usc.edu }}$ 


\begin{abstract}
Autoantibodies against SCLC-associated neuronal antigen ELAVL4 (HuD) have been linked to smaller tumors and improved survival, but the antigenic epitope and mechanism of autoimmunity have never been solved. We report that recombinant human ELAVL4 protein incubated under physiological conditions acquires isoaspartylation, a type of immunogenic protein damage. Specifically, the Nterminal region of ELAVL4, previously implicated in SCLC-associated autoimmunity, undergoes isoaspartylation in vitro, is recognized by sera from anti-ELAVL4 positive SCLC patients and is highly immunogenic in subcutaneously injected mice and in vitro stimulated human lymphocytes. Our data suggest that isoaspartylated ELAVL4 is the trigger for the SCLC-associated anti-ELAVL4 autoimmune response.
\end{abstract}

Keywords: small cell lung cancer; SCLC; autoimmunity; isoaspartylation 


\section{Introduction}

Small cell lung cancer (SCLC) is the most aggressive lung cancer subtype, with an overall 5-year survival of only $6 \%$ (Howlader et al., 2013). Aside from prophylactic cranial irradiation to limit brain metastases, there have been no meaningful therapeutic advances in three decades (Kalemkerian, 2014). The existence of multiple SCLC-associated autoimmune syndromes suggests an interplay between SCLC and the immune system. SCLC patients with autoimmune syndromes exhibit high titer antibodies against neuronal proteins ectopically expressed by their tumors and can show regression (Dalmau et al., 1992; Winter et al., 1993; Darnell and DeAngelis, 1993; Graus et al., 1997; Honnorat et al., 2009; Kobayashi et al., 2007; Kazarian and Laird-Offringa, 2011). Although the high titer patients are rare, the characteristic autoantibodies are found in a substantial fraction of SCLC patients without autoimmune symptoms, albeit at lower titers (Graus et al., 1997; Kazarian and Laird-Offringa, 2011; Dalmau et al., 1990). Thus, while an immune response is relatively common, it does not usually progress to a paraneoplastic disease. Autoantibodies in SCLC patients have been associated with improved survival, suggesting that the immune response could be harnessed therapeutically. Understanding the mechanism triggering SCLC-associated immune responses may provide new tools for SCLC detection, diagnosis and new treatments, such as immunotherapy (Kazarian and Laird-Offringa, 2011).

One well-known family of proteins that can become self antigens in SCLC is that of the neuronal embryonic lethal altered visual system-like (ELAVL) RNA-binding proteins (formerly "Hu" proteins), which are expressed in every SCLC, but not in non-SCLC (Manley et al., 1995). Antibodies to neuronal ELAVL correlate with improved survival of SCLC patients (Graus et al., 1997). Furthermore, in patients with full-blown anti-ELAVL autoimmune disease (paraneoplastic encephalomyelitis/sensory neuropathy, or PEM/SN), the tumors are often small and localized (Dalmau et al., 1992). Of the neuronal ELAVL proteins, tumors most commonly express ELAVL4 (HuD) (Manley et al., 1995). The 
highly homologous ELAVL2 (HuB/Hel-N1) and ELAVL3 (HuC) are also neuronal, while the less conserved ELAVL1 (HuR) is ubiquitously expressed. Although less than 1\% of SCLC patients develop high titer anti-ELAVL antibodies and exhibit PEM/SN, lower titer antibodies are seen in about 15-20\% of SCLC patients without autoimmune symptoms (Graus et al., 1997; Kazarian and Laird-Offringa, 2011; Dalmau et al., 1990). How this immune response develops remains in question. There is little evidence for immunogenic mutations (Sekido et al., 1994; Carpentier et al., 1998; D'Alessandro et al., 2010). Based on the sequence and presumably unstructured nature of the N-terminal region of neuronal ELAVL proteins, we hypothesized that in the context of SCLC these proteins can undergo isoaspartylation, a naturally-occurring immunogenic post-translational modification. Isoaspartyl moieties are normally repaired in the body, and abnormal isoaspartylation been implicated in several autoimmune diseases (Mamula et al., 1999; Yang et al., 2006). We hypothesized that it triggers an immune response in a subset of SCLC patients. Here we investigated the role of isoaspartylation in the anti-ELAVL response.

\section{Materials and Methods}

\section{Patients}

Sera were collected from Dutch patients with PEM/SN and/or SCLC in the University Hospital of Maastricht, the Netherlands, and were obtained with informed consent and approval by the medicalethical committees. We used sera previously determined to be positive for anti-ELAVL4 (“anti-Hu”) reactivity to gain a better understanding of the nature of the immune response. Use of the human serum samples was approved by the USC Institutional Review Board (protocol \# HS-10-00050).

Recombinant protein production, in vitro isoaspartyl (isoAsp) conversion and detection 
Similar to the N-terminal fragment used to broadly characterize the ELAVL immune epitopes in numerous studies (Manley et al., 1995; Sillevis Smit et al., 1996; Sodeyama et al., 1999; Graus et al., 1998), we produced recombinant human ELAVL4 (aa 1-117) polypeptide with a C-terminal hexahistidine tag. Recombinant ELAVL4 E-117 $_{1}$ was made in BL21(DE3) E. coli carrying a plasmid providing extra copies of a rare Arginine tRNA. Protein production was very high, so that high purity was achieved. In addition, all constructs carry the full RRM, forming a globular domain that folds well and that at purification is present in very high concentrations in solution. We measured endotoxin levels using the Pierce LAL Chromogenic Endotoxin Quantitation Kit (catalogue number 88282, Thermo Scientific, Rockford, IL) and levels were found to be negligible or undetectable. We used protein batches with undetectable endotoxin levels for experiments with human peripheral blood monocytes. ELAVL4 mutants were generated by site-directed mutagenesis and verified by sequencing. The mouse and human ELAVL4 N-terminal regions (aa 1-117) are identical except for a single conservative change (threonine 33 in human to alanine in mouse). Recombinant DNA work was carried out under BSL1 containment conditions. Proteins were incubated in $50 \mathrm{mM} \mathrm{K-HEPES} \mathrm{(pH} \mathrm{7.4),} 1.0 \mathrm{mM}$ EGTA, 0.02\% $(\mathrm{w} / \mathrm{v})$ sodium azide, and $5 \%(\mathrm{w} / \mathrm{v})$ glycerol) for up to 7 days at $37^{\circ} \mathrm{C}, 1 \mu \mathrm{g}$ per lane was resolved on protein gels and transferred to membranes for on-blot methylation with recombinant rat repair enzyme protein-L-isoaspartate O-methyltransferase (PIMT) and [ $\left.{ }^{3} \mathrm{H}\right]-\mathrm{S}$-adenosyl methionine(Zhu et al., 2006).

\section{Anti-isoAsp ELAVL4 antiserum generation and western blotting}

Rabbits were immunized with synthetically generated CTSNTS-isoAsp-GPSSNNR-amide peptide, conjugated to a carrier protein (YenZym Antibodies, San Francisco, CA). The antibody was affinitypurified using the isoAsp-containing peptide, then affinity-absorbed against the unmodified peptide. Specificity was analyzed by ELISA (YenZym Antibodies, see Fig. 2 A, B). The affinity-purified anti- 
isoAsp-ELAVL4 antibody (1:250) was used with secondary goat anti-rabbit IgG HRP conjugate $(1: 20,000)$ to detect isoaspartylation on western blots containing $0.25-1 \mu \mathrm{g}$ recombinant proteins, as indicated in the figure legends. For the peptide competition assay, $1 \mu \mathrm{g} / \mathrm{ml}$ of antibody was preincubated with 500-1000-fold molar excess of the native or isoAsp-containing ELAVL4 peptide at $4^{\circ} \mathrm{C}$ overnight. For detection of isoAsp conversion in brain lysates, $\sim 50 \mu \mathrm{g}$ of brain extract from PIMT wild type and knockout mice was subjected to western blot analysis with primary antibodies: rabbit antiisoAsp ELAVL4 (1:250); rabbit anti-actin (1:250, Cytoskeleton Inc., Denver, CO); mouse anti-human PIMT (1:1000, Santa Cruz Biotechnology, CA); rabbit anti-human ELAVL4 (1:250, Santa Cruz Biotechnology, CA). Secondary antibodies were added for one hour at room temperature with gentle agitation, either goat anti-rabbit IgG (1:20,000) or goat anti-mouse IgG (1:2000). Restore Plus Western Blot Stripping Buffer was used to strip and reprobe blots according to manufacturer's instructions (Thermo Scientific, Rockford, IL).

\section{Immunohistochemistry}

Serial $4 \mu \mathrm{m}$ thick sections of de-identified archival remnants of paraffin-embedded human SCLC tumor samples (National Disease Research Interchange, Philadelphia, PA) were subjected to haematoxylin/eosin staining or incubation with primary antibodies: rabbit anti-human ELAVL4 1:250 (Santa Cruz Biotechnology, Santa Cruz, CA); our rabbit anti-isoAsp ELAVL4 serum 1:400 (0.625 $\mu \mathrm{g} / \mathrm{ml}$ final concentration; YenZym, South San Francisco, CA); rabbit anti-human PIMT 1:1000 (Santa Cruz Biotechnology, Santa Cruz, CA). The EnVision+ System-HRP labeled polymer anti-rabbit (K4002, Dako North America, Inc., Carpinteria, CA) was visualized with liquid DAB chromogen (Biogenex, Fremont, CA), and ethyl-green applied as counter staining. Images were captured using the 
Aperio imaging system and processed using the compatible ImageScope viewing software. Sections were digitally analyzed in the range of $2 \mathrm{X}$ to $40 \mathrm{X}$ magnification.

\section{Mouse and human immunogenicity assays}

FVB/N mice were immunized subcutaneously with $150 \mu \mathrm{g}$ recombinant protein emulsified 1:1 with incomplete Freund's adjuvant and boosted at 7 days; spleens were harvested 10 days later. Blood was collected before immunization, just prior to boosting and at euthanasia. Plasma was tested for reactivity to native and isoAsp ELAVL4 using goat-anti-mouse IgG-HRP secondary antibody (1:2000). Due to very limited amounts of mouse blood, we first tested several different dilutions of the mouse plasma from preimmunized, pre-boosted and terminal bleed mice to determine which resulted in signal in the linear range. We then used the same dilution to tests all pre-immunization sera (1:2000), pre-boosting sera (1:2000) and terminal bleed plasma samples (1:40,000), using identical exposure times for all blots. We quantitated the blots using Bio-Rad imaging software, drawing a box around the bands in each lane and subtracting background activity for a similar size box in an area without signal. For mouse T-cell proliferation assays, single cell suspensions were prepared from harvested spleens. 300,000 cells were seeded in triplicate into pre-titrated 96-well culture plates containing serial dilutions of native or isoAsp-

converted ELAVL4 protein, vehicle, or positive control. Cells were pulsed with $0.5 \mathrm{mCi}\left[{ }^{3} \mathrm{H}\right]$ thymidine/well at 72 hours, incubated for 18 hours, harvested and counted using a TopCount Scintillation Counter (Perkin Elmer, Foster City, CA). Thymidine uptake in counts per minute (cpm) was determined by averaging replicate wells. All animal experiments were approved by the USC institutional animal care and use committee. Human peripheral blood mononuclear cells from unidentified donors were incubated for 3 days with $1 \mu \mathrm{g} / \mathrm{mL}$ native or isoAsp-ELAVL4 $4_{1-117}$, then pulsed with $\left[{ }^{3} \mathrm{H}\right]$ thymidine, incubated for $24 \mathrm{~h}$, harvested and counted. 


\section{ELAVL4 serum reactivity from anti-ELAVL4 positive SCLC-patients}

Serum samples from seven patients previously shown to exhibit an anti-ELAVL4 response were examined at dilutions ranging from 1:1000 to $1: 10,000$ depending on previously observed anti-ELAVL4 reactivity. Secondary antibody was HRP-conjugated anti-human $\operatorname{IgG}(1: 2,000)$.

\section{Statistical methods}

The student's two-sided t-test was used with a significance value of $p<0.05$.

\section{Results}

In vitro isoaspartyl conversion of ELAVL4

In vitro incubation under physiological conditions can be used to determine if a protein is highly susceptible to isoaspartylation (Johnson et al., 1989) (Fig. 1A). Recombinant human ELAVL4 was incubated at $\mathrm{pH} 7.4$ and $37^{\circ} \mathrm{C}$ for up to seven days. Initially, the experiment was performed with fulllength ELAVL4, which contains four potential canonical isoAsp-prone sites (amino acids D or N followed by $\mathrm{G}, \mathrm{S}$, or $\mathrm{H}$ in flexible regions of the protein). However, this protein quickly precipitated under the experimental conditions and was not used for any further experiments (data not shown). Because the N-terminal domain of ELAVL4 containing RNA recognition motif 1 (RRM1) had been strongly implicated in immune responses in SCLC patients (Manley et al., 1995; Sillevis Smit et al., 1996; Sodeyama et al., 1999; Graus et al., 1998), we proceeded with this segment of the protein (amino acids 1-117). The region N-terminal to RRM1 contains three potential canonical isoAsp conversion sites (N7, N15, and D36) and five additional $\mathrm{N}$ or D residues (Fig. 1B). Recombinant human ELAVL4 $4_{1-117}$

was incubated for $0,1,3$, and 7 days and tested for the presence of isoaspartylation by its ability to 
accept a radiolabeled methyl group (3H-S-adenosyl methionine) in an on-blot PIMT-catalyzed repair reaction (Fig. 1C). Native ELAVL4 $4_{1-117}$ exhibited little reactivity (Day 0), but the protein showed a strong increase in PIMT-mediated methylation over the incubation period. Mutation of the canonical isoAsp-prone sites N7, N15 and D36 only reduced labeling by about 25\% (not shown), indicating that additional sites are present. A mutant polypeptide fragment in which all eight $\mathrm{N}$ or $\mathrm{D}$ residues in the 38 aa N-terminal region had been mutated to $\mathrm{Q}$ or $\mathrm{E}$ (residues that are similar but not prone to isoaspartylation), did not appear to become isoaspartylated, nor did RRM1 alone (Fig. 1C). Thus we conclude that the region N-terminal to RRM1 becomes isoaspartylated in vitro under conditions of physiological $\mathrm{pH}$ and temperature.

In vivo isoaspartylation of ELAVL4

To determine whether isoaspartylation occurs in vivo, we raised a rabbit anti-isoAsp ELAVL4 antiserum. The region around the canonical isoaspartylation site $\mathrm{N}_{15}$ was determined to be a strong candidate for peptide generation based on its predicted antigenicity, hydrophilicity, secondary structure, and potential for post-translational events. Rabbits were immunized with a peptide containing an isoAsp residue at $\mathrm{N}_{15}$ and an N-terminal cysteine: CTSNTS-isoAsp-GPSSNNR (Fig. 1B, underlined). Serum was collected and affinity purified on a column carrying the isoAsp-containing peptide, followed by affinity absorption of non-isoAsp-specific reactivity on a native peptide column (Fig. 2A). Whereas the antibody purified from the first column recognized both the native and isoAsp forms of the peptide, following immuno-absorption, the antibody showed considerable specificity for the isoAsp-containing ELAVL4 peptide as demonstrated by ELISA (Fig. 2B). To verify the ability of the affinitypurified/absorbed antibody to specifically recognize isoAsp-containing ELAVL4, we examined its reactivity against ELAVL4 $4_{1-117}$, a single $\mathrm{N}_{15} \mathrm{Q}$ mutant and an $\mathrm{N}_{7} \mathrm{Q}+\mathrm{N}_{15} \mathrm{Q}$ double mutant, all incubated 
under isoaspartylation-inducing conditions as in Figure 1C. The double mutant was generated because the protein sequence in the $\mathrm{N}_{15}$ region is partially homologous to the region containing $\mathrm{N}_{7}$, and the antiserum could therefore be cross-reactive with this region (see Fig. 1B, starred). Western blot analysis using the purified anti-isoAsp ELAVL4 antiserum showed some reactivity to wild type protein at day 0 , possibly due to residual cross reactivity of the antiserum with the unconverted peptides or to limited isoAsp conversion during protein production (Fig. 2C). However, over time, ELAVL4 ${ }_{1-117}$ showed a dramatic increase in reactivity. Additionally, during the incubation period reactivity appeared against bands of higher molecular weight, which seem to be multimers of the protein that are not disaggregated despite denaturing conditions. Isoaspartylation has been implicated in multimerization and aggregation (Shimizu et al., 2000; Kern et al., 2005; Zirah et al., 2006; Paranandi and Aswad, 1995). The ELAVL4 ${ }_{1-}$ 117 multimers are barely visible on Coomassie gels but do become visible during the on-blot methylation (Fig. 1C), suggesting that they contain isoAsp sites and are substrates for repair by PIMT.

The $\mathrm{N}_{15} \mathrm{Q}$ and $\mathrm{N}_{7} \mathrm{Q}+\mathrm{N}_{15} \mathrm{Q}$ mutant proteins showed weaker reactivity with the rabbit antiserum than the wild type protein, suggesting that the antiserum is specific for the $\mathrm{N}_{15}$ site (Fig. 2C). However, some reactivity accumulated over time and the higher molecular weight complex still showed substantial signal, suggesting that the aggregate is highly reactive with the antibodies. To further examine the isoAsp specificity of the antibody, we incubated the antiserum with a 1000-fold molar excess of either the native or isoAsp-containing ELAVL4 peptide (Fig. 2D). While the native peptide did not compete, the isoAsp peptide almost completely prevented recognition of in vitro isoAsp-converted ELAVL4 $4_{1-117}$ protein, confirming ELISA data indicating that the antiserum shows considerable specificity for the post-translational modification. We also tested the antiserum for cross reactivity with the other ELAVL family proteins and observed reactivity with the two other neuronal ELAVL family members (Pulido et al, 2016). The highly conserved ELAVL2 (HelN1/HuB) showed a strong interaction and ELAVL3 
$(\mathrm{HuC})$ a weaker one. In contrast, the antisera did not react with the ubiquitously expressed ELAVL1 (HuR).

To determine whether isoaspartylation of neuronal ELAVL proteins occurs in vivo, we used the affinity purified anti-isoAsp-ELAVL4 rabbit antiserum to examine brain lysates from PIMT knockout mice, which are known to accumulate high levels of isoAsp-containing proteins in the brain, a place where PIMT is highly expressed (Zhu et al., 2006; Kim et al., 1997; Yamamoto et al., 1998). The isoAsp-ELAVL4-specific serum was much more reactive with lysates from PIMT knockout mice than control animals (Fig. 3A, B), indicating that neuronal ELAVL proteins can undergo isoaspartylation in vivo.

We next used the anti-isoAsp-ELAVL4 rabbit antiserum to examine human SCLC tumors; neuronal ELAVL proteins are among many abnormally expressed neuronal proteins observed in SCLC tumors (Manley et al., 1995) and might accumulate isoaspartylation due to ineffective repair of spontaneously isoaspartylated protein. Because SCLC patients are rarely operated, paraffin-embedded tumor samples are rare (most diagnoses are made by fine needle aspiration). We obtained and analyzed 5 paraffin-embedded tumors; as expected all showed high anti-ELAVL4 reactivity (Manley et al., 1995; Dalmau et al., 1992) as well as reactivity with the anti-isoAsp-ELAVL4 antiserum (Fig. 3C, and data not shown). Competition with ELAVL4 $4_{1-117}$ incubated under isoaspartylation conditions but not native ELAVL4 $4_{1-17}$ depleted reactivity with the isoAsp-specific antiserum, supporting the specificity of the assay. Probing of adjacent sections with an antiserum to PIMT showed little staining. PIMT is most highly expressed in the nervous system and is normally present but low in the lung. Our results strongly support the presence of neuronal isoAsp-ELAVL proteins in SCLC. A larger picture of the images is included in the supplementary data (Supplementary Fig. S1). 


\section{Immunogenicity of isoaspartylated ELAVL4 ${ }_{1-117}$}

To test the effect of isoaspartylation on the immunogenicity of the ELAVL4 $4_{1-117}$ fragment, we immunized mice subcutaneously with the native ELAVL $4_{1-117}$ fragment or protein that had been incubated under isoAsp-inducing conditions for 7 days. Mice were boosted after one week and euthanized 10 days later. B- and T-cell responses were evaluated (Fig. 4A). Mice immunized with isoAsp-converted protein showed an early and strong antibody response even without a booster injection (Fig. 4B, C). As expected based on previous immunization experiments with isoAsp-containing peptides (Mamula et al., 1999), antisera reacted with both native and isoaspartylated protein. IsoAsp-ELAVL4 ${ }_{1}$ 117 immunized mice also showed a stronger T-cell response than mice that had been immunized with native ELAVL4 ${ }_{1-117}$ (Fig. 4D).

To corroborate these results on human immune cells, we incubated human peripheral blood mononuclear cells (PBMC) with native or isoaspartylated ELAVL4 $4_{1-117}$, pulsed them with $\left[{ }^{3} \mathrm{H}\right]$ thymidine after three days, and measured cell proliferation 1 day later. Cell proliferation in PBMC treated with isoAsp-ELAVL4 $4_{1-117}$ increased significantly compared to PBMC treated with native ELAVL4 $_{1-117}$ (Fig. 4E). Thus, as in the mouse, human immune cells appear to respond more strongly to isoaspartylated ELAVL4 ${ }_{1-117}$, supporting the idea that unrepaired isoAsp-ELAVL4 could trigger immune responses in SCLC patients. In all assays, the difference in immune response to native $v s$. isoAsp protein was likely underestimated because the native protein undergoes spontaneous isoaspartylation during the immunization/incubation periods.

Sera from anti-ELAVL-positive SCLC patients react with the isoAsp-prone $N$-terminal region

If isoaspartylation causes anti-ELAVL reactivity in SCLC, we should detect reactivity against the N-terminal isoAsp-prone region of ELAVL4 using patient antisera. We examined 7 antisera from 
patients that had been previously confirmed to show anti-ELAVL activity. Three sera were from patients with the anti-ELAVL ("anti-Hu") syndrome PEM/SN and neurological symptoms, one from a SCLC patient with anti-ELAVL response but a different paraneoplastic disease, Lambert Eaton myasthenic syndrome (LEMS), and three from SCLC patients with a positive but weaker immune response and no neurological symptoms (Table 1). The latter type of patients is much more common than patients with high titer antibodies (Graus et al., 1997; Kazarian and Laird-Offringa, 2011; Dalmau et al., 1990).

Sera were tested by western blot for reactivity with ELAVL4 $4_{1-117}$ or RRM1 alone (we were unable to produce ELAVL4 $4_{1-38}$ because it precipitated), both incubated under isoaspartylation-inducing conditions (Fig. 5). As with the rabbit antiserum, the human sera reacted strongly with isoaspartylated protein and higher molecular weight complexes of ELAVL4 $4_{1-117}$. Sera from SCLC patients previously shown to be negative for anti-ELAVL4 reactivity lacked reactivity with both native and isoAspELAVL4 (Supplementary Fig. S2). It is remarkable that rabbits immunized with a short synthetic isoaspartylated ELAVL4 peptide generated an immune response so similar to that of human SCLC patients, whose exposure to ELAVL4 is linked to the aberrant expression of the protein in their tumor. This further supports the notion that the patient antigenic response is linked to isoaspartylation. The human sera also reacted with native protein (Day 0), a phenomenon frequently observed when isoAspcontaining peptides are used to immunize animals, and attributed to epitope spreading (Mamula et al., 1999; Mamula, 1998). Notably, all sera we tested from patients with moderate to strong ELAVL4 reactivity showed weak or no reactivity with the RRM1 domain alone, indicating that the epitope(s) against which the patient antibodies are directed are located in the 38-amino acid N-terminal region (Fig. 5 and data not shown).

\section{Discussion}


For over two decades it has remained unknown why SCLC patients exhibit unusual immune responses to neuronal proteins that are abnormally expressed in their tumors. Here, we studied the antiELAVL4 response to solve this enigma. Previous experiments using recombinant protein and deletion mutants using numerous SCLC patient antisera known to be anti-ELAVL positive have consistently pointed to the N-terminal portion of the protein which carries RRM1 (Manley et al., 1995; Sillevis Smit et al., 1996; Sodeyama et al., 1999; Graus et al., 1998). This 117-amino acid N-terminal protein fragment showed reactivity with every patient serum sample tested. The identical region was identified using sera from 7 transgenic mice with SCLC that were anti-ELAVL4 positive (Kazarian et al., 2009). However, none of these experiments examined RRM1 in isolation. We consistently observed that reactivity against RRM1 alone was much weaker or absent in sera from SCLC patients despite an antibody response to the full 117 -amino acid fragment. This suggests that the 38 -amino acid region Nterminal to RRM1 plays a key role in the SCLC-associated autoimmune response. A previous epitope mapping experiment using the $\mathrm{N}$-terminal amino acids fused to a partial RRM1 did not detect reactivity with SCLC patient antisera (Sodeyama et al., 1999). However, the authors did not demonstrate successful purification of the recombinant protein fragment and we have found that effective production of the N-terminal piece requires the globular RRM, possibly as a solubility "handle". Combined with our demonstration that ELAVL4 becomes isoaspartylated in vitro and in vivo, the well-documented immunogenicity of isoaspartylated proteins and peptides (Yang et al., 2006; Doyle et al., 2003), and our demonstrated immunogenicity of isoaspartylated ELAVL4 $4_{1-117}$, the data make a strong case for isoaspartylation of neuronal ELAVL proteins as the trigger for SCLC-associated anti-ELAVL reactivity. Isoaspartylation happens naturally under physiological conditions and the repair enzyme PIMT is expressed in all organisms from bacteria to mammals. While PIMT is present in all mammalian tissues, it is most prominent in the brain, retina, and testis (Diliberto and Axelrod, 1976; Mizobuchi et al., 1994; 
Qin et al., 2014). PIMT is crucial for normal neuronal activity; mice lacking the enzyme show a dramatic increase in protein isoaspartylation in the brain and die of seizures a few weeks after birth (Kim et al., 1997; Yamamoto et al., 1998; Desrosiers and Fanelus, 2011). This would suggest that neuronal proteins, either through their function or location, are more prone to isoAsp conversion. Misexpression of neuronal proteins in non-neuronal types of cells, such as SCLC tumors, might lead to the accumulation of isoaspartylated proteins. PIMT levels in SCLC may not be as high as in the nervous system (we detected little if any reactivity with PIMT antibodies in SCLC tumors), and conditions such as stress, hypoxia and/or necrosis might favor isoaspartylation. It is also possible that the repair enzyme might not be able to access damaged protein spilling from necrotic cells. Thus, the amount of isoaspartylated protein to which SCLC patients are exposed might vary based on the level of expressed PIMT, the amount of necrosis, or other factors, such as single nucleotide polymorphisms that are known to affect PIMT efficacy (Qin et al., 2014; Desrosiers and Fanelus, 2011). Exposure to sufficient levels of isoaspartylated ELAVL proteins to trigger an immune response might be a stochastic event and may not happen in all SCLC patients, even if all SCLC tumors express neuronal ELAVL family members. Indeed, in an inbred transgenic SCLC mouse model, despite ubiquitous expression of neuronal ELAVL proteins in the cancers, only $14 \%$ of mice developed a detectable anti-ELAVL response(Kazarian et al., 2009).

Based on our data with ELAVL4, one would expect isoaspartylation to play a role in paraneoplastic responses to other SCLC-associated antigens. Well over 50 neuronal proteins (many as yet uncharacterized) undergo isoaspartylation in the brains and retinas of PIMT knockout mice (Zhu et al., 2006; Qin et al., 2014). Two of these, alpha-enolase and recoverin, are indeed antigens in SCLCassociated retinopathy (Adamus et al., 1996). Interestingly, the paraneoplastic retinopathy-associated antigens are not only misexpressed in SCLC, but also in breast cancer, melanoma and gynecological 
cancers, all tissues that would normally not express neuronal proteins (Adamus, 2009). Thus, the findings of our study may have implications for numerous types of cancer. Examination of the sequence and predicted structure of known cancer-associated antigens reveals many potential isoaspartylation sites. For example, cerebellar degeneration-related protein 2 (CDR2), targeted by characteristic "antiYo" antibodies mainly in ovarian cancer patients, carries numerous potential isoaspartylation sites in the regions linking the known protein domains. Just like ELAVL4 in SCLC, the CDR2 antigen was present in all tested ovarian tumors, but only a quarter of patients carry anti-Yo antibodies (Totland et al., 2011). Isoaspartylation of neuronal antigens might also occur in non-cancer patients. For example, in at least one dementia patient with anti-NMDA receptor autoantibodies, the antigenic site lay in a loop containing an NG sequence - a typical isoaspartylation-prone sequence - and is abolished by the N368Q mutation (Doss et al., 2014), which prevents isoaspartylation. Investigating the propensity of neuronal antigens to undergo isoaspartylation in SCLC as well as other disease states will be important, but may be challenging due to the tendency of proteins to aggregate or precipitate under isoaspartylation conditions. Studies will likely require tailored isoAsp-specific antibodies such as those raised by us. The antiserum we raised was context-dependent and did not react with other isoaspartylated proteins (Fig. 2C and unpublished data). A generic isoAsp-reactive antibody would be very useful but it may be difficult to generate as the isoAsp epitope is shallow and embedded in the flanking protein sequence (Griffith et al., 2001).

Our data suggest that isoaspartylation of ELAVL4 and other neuronal antigens is the trigger for SCLC-associated autoimmune responses. Isoaspartylated antigens could be powerful new cancerspecific targets for immunotherapy in SCLC. It was shown in a mouse model that isoAsp-conversion of melanoma antigen TRP-2 (tyrosinase-related protein-2) resulted in the generation of a T-cell response in vitro and in vivo in which $\mathrm{CD} 8+\mathrm{T}$ cells were recruited to melanoma, and autoantibodies capable of 
binding tumor cells were produced (Doyle et al., 2006). Tumor growth was delayed upon isoAsp TRP-2 immunization, perhaps influencing immunologic clearance of the tumor. Indeed, anti-ELAVL4 autoantibodies in SCLC patients have been correlated with limited disease, complete response to therapy and improved survival (Graus et al., 1997) and have been associated with anecdotal spontaneous regression (Darnell and DeAngelis, 1993). Our mouse immunization experiments show that strong Band T-cell responses against ELAVL4 can be elicited with isoaspartylated protein and provide preclinical evidence for the feasibility of immunotherapy. It is intriguing that mice immunized with the native protein do not show a T-cell response but do develop antibodies after 17 days. One challenge of immunization experiments with native protein remains the possibility that proteins may undergo conversion and aggregation during the experiment. Since isoAsp moieties are known to aggregate, it is possible that the aggregates might form in the immunized location and might trigger a partially $\mathrm{T}$-cell independent response, which might explain the lack of T-cell response seen in the case of the native protein. The structure of isoAsp aggregates is unknown and how such aggregates, not only of ELAVL4 but of other isoasprtylated proteins might play a role in directing the immune response is a very interesting question. The study of immune triggering by isoaspartylated proteins is still in its infancy.

In considering the use of isoaspartylated proteins for immunotherapy it will of course be of crucial importance to carefully evaluate the safety of such immunizations to avoid potential deleterious consequences, such as inducing autoimmunity and neurological disease. In rare cases, severe autoimmune anti-ELAVL responses in SCLC patients result in acute neurological symptoms and death. However, previous studies in which animals were immunized with native ELAVL4 did not yield any neurological symptoms (Sillevis Smitt et al., 1995) and SCLC patients with substantial antibody titers but no autoimmune symptoms have been noted(Monstad et al., 2004). Therapeutic efficacy of ELAVL4 immunization has been suggested when mice were implanted with simulated SCLC cells (cells 
transfected with ELAVL4, (Ohwada et al., 1999)). Further experiments using a transgenic SCLC mouse model system will be very informative, as these mice accurately mimic human SCLC, including the anti-ELAVL4 response (Kazarian et al., 2009). Problems with epitope spreading could be avoided by using isoAsp-specific monoclonal antibodies as therapy as opposed to immunization with isoaspartylated protein, although recruitment of the patient's own immune system also provides a cellular response and would potentially provide a stronger and more long-term protection. The identification of isoAsp sites as potential new targets for SCLC detection, diagnosis, and/or treatment provides hope in a deadly disease that has seen minimal therapeutic advances in the last 30 years. 


\section{Acknowledgements}

Dedicated in loving memory to Carole Auerbach. We thank members of the Laird-Offringa lab for helpful comments and discussion.

\section{Grant Support}

The Auerbach, Pembroke and Mettler families, V Foundation (003696-00001), Department of Defense (W81XWH-10-1-0622) (ILO); NIH (NS-17269, DWA); USC Provost's Undergraduate Research Fellowship (EMC); Southern California Clinical and Translational Science Institute (TL1TR000132, MAP, AWW); Walter A. Richter Cancer Research Chair (WMK); Norris Comprehensive Cancer Center Core Grant (NIH/NCI P30CA014089). The funding agencies of the study had no role in study design, data collection, data analysis, data interpretation, or writing of the manuscript.

\section{FIGURE LEGENDS}

Fig. 1. ELAVL4 N-terminal region becomes isoaspartylated in vitro. (A) Mechanism of isoAsp formation and repair. Isoaspartylation occurs by spontaneous dehydration or deamidation of an asparagine (Asn-Xaa) or aspartate (Asp-Xaa) linkage (where Xaa is usually glycine, serine or histidine). This produces a metastable succinimide intermediate, which in turn undergoes spontaneous hydrolysis to generate an unequal mixture, typically $15-30 \%$ of the normal L-aspartyl linkage and $70-85 \%$ of an abnormal L-isoaspartyl-Xaa linkage with a kinked protein backbone (protein backbone indicated in black). PIMT converts L-isoaspartyl sites to $\alpha$-carboxyl-O-methyl esters, converting methyl donor S- 
adenosyl methionine (SAM) to S-adenosyl homocysteine (SAH). At physiological $\mathrm{pH}$ and temperature, the $\alpha$-carboxyl-O-methyl esters have a typical half-life of a few minutes and undergo spontaneous demethylation to reform the succinimide intermediate. Each PIMT cycle typically repairs $\sim 25 \%$ of the isoAsp peptide bond by converting it to a normal aspartyl peptide. Unrepaired peptides are continuously recycled, and the overall repair efficiency is $85 \%$ or greater. (B) Canonical isoAsp sites ( $\mathrm{N}$ or D followed by $\mathrm{S}, \mathrm{G}$ or $\mathrm{H}$ in unstructured regions) are boxed, all $\mathrm{N}$ and $\mathrm{D}$ with potential for isoaspartylation are bolded. It is unlikely that the globular structure of the RRM1 domain (dashed box) allows isoaspartylation of RRM1 residues. The isoAsp-containing peptide used to immunize rabbits is underlined and homologous amino acids shared between this isoAsp-containing peptide and the peptide sequence surrounding N7 are starred; (C) Recombinant wild type ELAVL4 ${ }_{1-117}$ protein, a mutant with substitutions at all $\mathrm{N}$ (to Q) and $\mathrm{D}$ (to E) residues in the $\mathrm{N}$-terminal 38 amino acids (indicated by the dashed line), and ELAVL439-117, a protein without the N-terminal region, were incubated at physiological conditions for 0,3 , and 7 days, separated by gel electrophoresis, transferred to a membrane and subjected to on-blot labeling with PIMT and ${ }^{3}$ H-SAM (top panel). Only wild type ELAVL4 $4_{1-117}$ showed above background labeling by PIMT at 3 and 7 days. The bottom panel shows a Coomassie-stained gel, indicating similar loading of wild type, mutant, and truncated proteins over the incubation period. A representative image of several similar experiments is shown.

Fig. 2. An anti-isoAsp ELAVL4 antiserum detects isoaspartylation in vitro

(A) Schematic representation of polyclonal rabbit anti-isoAsp ELAVL4 antiserum purification. The labels a-d match those in the histogram in panel B. (B) ELISA analysis of pre-purified serum (a, 1:100,000 dilution), affinity purification flow-through (b, 1:100,000 dilution), affinity purified eluted antibody (c, $0.01 \mu \mathrm{g} / \mathrm{ml}$ ), affinity-absorbed antibody (d, $0.01 \mu \mathrm{g} / \mathrm{ml}$ ), and no antibody controls (leftmost 
white and grey bars) against an unmodified ELAVL4 peptide and the same peptide containing an isoaspartate residue at $\mathrm{N}_{15}$ (see Figure 1B, underlined). ELISA data provided by YenZym Antibodies, South San Francisco, CA. (C) The isoAsp-ELAVL4 antisera specifically recognizes in vitro isoAspconverted ELAVL4 ${ }_{1-117 .} 2 \mu \mathrm{g}$ wild type, single mutant $\mathrm{N}_{15} \mathrm{Q}$ and double mutant $\mathrm{N}_{7} \mathrm{Q}+\mathrm{N}_{15} \mathrm{Q}$ protein per lane were in vitro isoaspartyl converted at $37^{\circ} \mathrm{C}$ for up to seven days. Samples from day $0,1,3$, and 7 were subjected to SDS-PAGE and Western blot analysis and exposed for 5 minutes. A representative example of triplicate experiments is shown. (See Pulido et al. 2016 for reactivity of antiserum with other ELAVL family members.) (D) Competition assay using the same ELAVL4 peptide against which the rabbit antisera had been raised, in its native or isoaspartylated form.

Fig. 3. Small cell lung cancer antigen ELAV is prone to isoaspartylation in vivo. (A) Brain extract from PIMT wild type and knock out mice was subjected to Western blot analysis. The brain is the normal site of ELAVL4 expression. Blots were probed with anti-PIMT and anti-ELAVL4 antibodies and the rabbit affinity-purified anti-isoAsp ELAVL4 antiserum. Membranes were stripped and reprobed using an antiactin antibody to check for similar loading. Exposure time was 45 seconds. (B) Quantitation of isoAspELAVL4 reactivity. (C) Anti-ELAVL4 reactivity of human archival SCLC tumors. Two representative cases are shown out of 5 examined. De-identified archival paraffin blocks were sectioned and subjected to H\&E staining or immunohistochemistry with antisera to ELAVL4, isoAsp-ELAVL4, and PIMT. Specificity of the anti-ELAVL4 antiserum was assessed by competition with excess native or isoAspconverted ELAVL4 $4_{1-117}$. Asterisks and circles are provided for sample orientation purposes. Top panel, magnification of sections 10X; lower panel, 20x. 
Fig. 4. Isoaspartylated ELAVL4 ${ }_{1-117}$ is highly immunogenic. (A) Schematic depicting strategy to assess the immunogenic potential of isoaspartylated-ELAVL4 in mice. Three mice each, immunized with native ELAVL4 $4_{1-11}$, the same protein incubated under isoaspartylation conditions for 7 days, or PBS were used. (B) Anti-ELAVL4 and anti-isoAsp-ELAVL4 reactivity of mouse plasma was determined as in Fig. 2C by western blot analysis using $0.25 \mu \mathrm{g}$ recombinant protein in its native form (Nat) or incubated under isoapsartylation conditions for 7 days (Iso); dilutions of mouse plasma are indicated at top (post-boosting sera were more diluted). The right-most panel shows rehybridization of the strips from mouse 525 with an anti-(His) 6 tag antibody. (C) Quantification of mouse antibody response. (D) Tcell proliferation comparing mice immunized with native vs. isoAsp-ELAVL4 1 -117. (E) Induction of

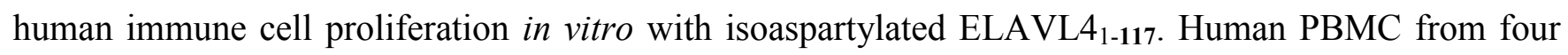
unidentified donors were incubated in triplicate with native ELAVL4 ${ }_{1-117}$, isoAsp-ELAVL4 ${ }_{1-117}$ or positive control phytohemagglutinin (PHA) $72 \mathrm{~h}$ prior to $\left[{ }^{3} \mathrm{H}\right]$ thymidine pulse. Total cell proliferation was then measured at $96 \mathrm{~h}$. This graph is a representative example of an experiment. $* \mathrm{p}<0.05, * * *$ $\mathrm{p}<0.001$.

Fig. 5. SCLC patient sera target the isoAsp-prone region. Recombinant ELAVL4 $4_{1-117}$ and RRM1 alone were subjected to isoAsp-inducing conditions and gel electrophoresis. The top left panel shows a Coomassie stain of a representative gel. As the protein acquires isoaspartylation (Day 3, 7), mobility changes, widening the band on gel. The other panels show blots probed with patient antiserum (top section) or stripped and reprobed with an anti-(His) 6 antibody $(1: 10,000-20,000)$ recognizing the Cterminal hexahistidine tag on the recombinant proteins (only bottom section of each panel is shown to indicate protein presence). Top row: 1:10,000 human serum dilution. Bottom row 1:1,000 human serum 
dilution, except for patient 7, 1:500 dilution. Note the weak or absent staining of the RRM1 alone fragment with the human sera. 
Table 1. Properties of anti-ELAVL-positive patients ${ }^{1}$

\begin{tabular}{|l|l|l|l|l|l|l|l|l|}
\hline Patient & $\begin{array}{l}\text { Paraneoplastic } \\
\text { Disease }\end{array}$ & Gender & $\begin{array}{l}\text { Age at } \\
\text { diagnosis }\end{array}$ & $\begin{array}{l}\text { Smoking } \\
\text { History }\end{array}$ & $\begin{array}{l}\text { Tumor and } \\
\text { stage }\end{array}$ & $\begin{array}{l}\text { Age } \\
\text { deceased }\end{array}$ & $\begin{array}{l}\text { Survival } \\
\text { months })\end{array}$ & $\begin{array}{l}\text { Initial response to } \\
\text { treatment }\end{array}$ \\
\hline 1 & PEM/SN & F & 72 & Unknown & $\begin{array}{l}\text { SCLC, } \\
\text { LD }\end{array}$ & 72 & 0 & No treatment \\
\hline 2 & PEM/SN & F & 66 & $\begin{array}{l}50 \text { pack- } \\
\text { years }\end{array}$ & $\begin{array}{l}\text { n/a; no } \\
\text { SCLC or } \\
\text { other } \\
\text { tumor }\end{array}$ & 76 & n/a & n/a \\
\hline 3 & PEM/SN & F & 43 & Unknown & $\begin{array}{l}\text { Primary } \\
\text { unclear, } \\
\text { ED }\end{array}$ & 45 & 21 & $\begin{array}{l}\text { Complete } \\
\text { response }\end{array}$ \\
\hline 4 & LEMS & M & 76 & 75 pack- & $\begin{array}{l}\text { SCLC, } \\
\text { LD }\end{array}$ & 84 & 99 & $\begin{array}{l}\text { Complete } \\
\text { response }\end{array}$ \\
\hline 5 & none & F & 65 & Unknown & SCLC, & 66 & 10 & $\begin{array}{l}\text { Complete } \\
\text { response }\end{array}$ \\
\hline 6 & none & M & 74 & Unknown & $\begin{array}{l}\text { SCLC, } \\
\text { LD }\end{array}$ & 74 & 0 & No response \\
\hline 7 & none & F & 55 & $\begin{array}{l}\text { Previous } \\
\text { smoker }\end{array}$ & $\begin{array}{l}\text { SCLC, } \\
\text { LD }\end{array}$ & 56 & 8 & No response \\
\hline
\end{tabular}

1. Patients 1-3 were diagnosed with PEM/SN, which is typically associated with anti-ELAVL

antibodies; patient 2 showed no detectable tumor in 11-year follow up despite suspected SCLC; patient

3 showed neuroendocrine brain metastasis but no primary tumor at the time -origin of metastasis was

unclear since she had a history of ovarian carcinoma and pulmonary metastasis; patient 4 showed anti-

ELAVL4 antibodies but exhibited LEMS, which is associated with antibodies against voltage-gated

calcium channels; patients 5-7 showed no paraneoplastic autoimmune neurological disease. LD: limited disease, ED: extensive disease. 


\section{REFERENCES}

Adamus, G. (2009) Autoantibody targets and their cancer relationship in the pathogenicity of paraneoplastic retinopathy. Autoimmun Rev, 8, 410-414.

Adamus, G. et al. (1996) The occurrence of serum autoantibodies against enolase in cancer-associated retinopathy. Clin Immunol Immunopathol, 78, 120-129.

Carpentier, A.F. et al. (1998) Absence of HuD gene mutations in paraneoplastic small cell lung cancer tissue. Neurology, 50, 1919.

D'Alessandro, V. et al. (2010) Molecular analysis of the HuD gene in neuroendocrine lung cancers. Lung Cancer, 67, 69-75.

Dalmau, J. et al. (1992) Anti-Hu--associated paraneoplastic encephalomyelitis/sensory neuronopathy. A clinical study of 71 patients. Medicine (Baltimore), 71, 59-72.

Dalmau, J. et al. (1990) Detection of the anti-Hu antibody in the serum of patients with small cell lung cancer--a quantitative western blot analysis. Ann. Neurol.Ann Neurol, 27, 544-552.

Darnell, R.B. \& DeAngelis, L.M. (1993) Regression of small-cell lung carcinoma in patients with paraneoplastic neuronal antibodies. Lancet, 341, 21-22.

Desrosiers, R.R. \& Fanelus, I. (2011) Damaged proteins bearing L-isoaspartyl residues and aging: a dynamic equilibrium between generation of isomerized forms and repair by PIMT. Curr Aging Sci, 4, 818.

Diliberto, E.J.J. \& Axelrod, J. (1976) Regional and subcellular distribution of protein carboxymethylase in brain and other tissues. Journal of Neurochemistry, 26, 1159-1165.

Doss, S. et al. (2014) High prevalence of NMDA receptor IgA/IgM antibodies in different dementia types. Ann Clin Transl Neurol, 1, 822-832. 
Doyle, H.A. et al. (2003) A failure to repair self-proteins leads to $\mathrm{T}$ cell hyperproliferation and autoantibody production. Journal of Immunology, 171, 2840-2847.

Doyle, H.A. et al. (2006) Isoaspartyl post-translational modification triggers anti-tumor T and B lymphocyte immunity. Journal of Biological Chemistry, 281, 32676-32683.

Graus, F. et al. (1997) Anti-Hu antibodies in patients with small-cell lung cancer: association with complete response to therapy and improved survival. Journal of Clinical Oncology, 15, 2866-2872.

Graus, Y.F. et al. (1998) Selection of recombinant anti-HuD Fab fragments from a phage display antibody library of a lung cancer patient with paraneoplastic encephalomyelitis. Journal of Neuroimmunology, 82, 200-209.

Griffith, S.C. et al. (2001) Crystal structure of a protein repair methyltransferase from Pyrococcus furiosus with its L-isoaspartyl peptide substrate. Journal of Molecular Biology, 313, 1103-1116.

Honnorat, J. et al. (2009) Onco-neural antibodies and tumour type determine survival and neurological symptoms in paraneoplastic neurological syndromes with $\mathrm{Hu}$ or CV2/CRMP5 antibodies. J Neurol Neurosurg Psychiatry, 80, 412-416.

Howlader, N. et al. (2013) SEER Cancer Statistics Review, 1975-2011. National Cancer Institute. Bethesda, MD, http://seer.cancer.gov/csr/1975_2011/, based on November 2013 SEER data submission, posted to the SEER web site, April 2014.

Johnson, B.A. et al. (1989) Formation of isoaspartate at two distinct sites during in vitro aging of human growth hormone. Journal of Biological Chemistry, 264, 14262-14271.

Kalemkerian, G.P. (2014) Chemotherapy for small-cell lung cancer. Lancet Oncol, 15, 13-14.

Kazarian, M. et al. (2009) Immune response in lung cancer mouse model mimics human anti-Hu reactivity. Journal of Neuroimmunology, 217, 38-45.

Kazarian, M. \& Laird-Offringa, I.A. (2011) Small-cell lung cancer-associated autoantibodies: potential applications to cancer diagnosis, early detection, and therapy. Molecular Cancer, 10, 33. 
Kern, R. et al. (2005) Protein isoaspartate methyltransferase is a multicopy suppressor of protein aggregation in Escherichia coli. Journal of Bacteriology, 187, 1377-1383.

Kim, E. et al. (1997) Deficiency of a protein-repair enzyme results in the accumulation of altered proteins, retardation of growth, and fatal seizures in mice. Proceedings of the National Academy of Sciences of the United States of America, 94, 6132-6137.

Kobayashi, M. et al. (2007) Long-term survival of a patient with small cell lung cancer associated with cancer-associated retinopathy. Lung Cancer, 57, 399-403.

Mamula, M.J. (1998) Epitope spreading: the role of self peptides and autoantigen processing by B lymphocytes. Immunological Reviews, 164, 231-239.

Mamula, M.J. et al. (1999) Isoaspartyl post-translational modification triggers autoimmune responses to self-proteins. Journal of Biological Chemistry, 274, 22321-22327.

Manley, G.T. et al. (1995) $\mathrm{Hu}$ antigens: reactivity with $\mathrm{Hu}$ antibodies, tumor expression, and major immunogenic sites. Annals of Neurology, 38, 102-110.

Mizobuchi, M. et al. (1994) Tissue-specific expression of isoaspartyl protein carboxyl methyltransferase gene in rat brain and testis. Journal of Neurochemistry, 62, 322-328.

Monstad, S.E. et al. (2004) Hu and voltage-gated calcium channel (VGCC) antibodies related to the prognosis of small-cell lung cancer. Journal of Clinical Oncology, 22, 795-800.

Ohwada, A. et al. (1999) DNA vaccination against HuD antigen elicits antitumor activity in a small-cell lung cancer murine model. American Journal of Respiratory Cell and Molecular Biology, 21, 37-43.

Paranandi, M.V. \& Aswad, D.W. (1995) Spontaneous alterations in the covalent structure of synapsin I during in vitro aging. Biochemical and Biophysical Research Communications, 212, 442-448.

Pulido, M.A., DerHartunian, M.K., Sehgal, P. \& Laird-Offringa, I.A. (2016) Neuronal ELAVL proteins are prone to isoaspartylation. Manuscript submitted to Data in Brief. 
Qin, Z. et al. (2014) Isoaspartyl protein damage and repair in mouse retina. Invest Ophthalmol Vis Sci, 55, 1572-1579.

Sekido, Y. et al. (1994) Molecular analysis of the HuD gene encoding a paraneoplastic encephalomyelitis antigen in human lung cancer cell lines. Cancer Research, 54, 4988-4992.

Shimizu, T. et al. (2000) Isoaspartate formation and neurodegeneration in Alzheimer's disease. Archives of Biochemistry and Biophysics, 381, 225-234.

Sillevis Smit, P. et al. (1996) The HuD paraneoplastic protein shares immunogenic regions between PEM/PSN patients and several strains and species of experimental animals. Journal of Neuroimmunology, 71, 199-206.

Sillevis Smitt, P.A. et al. (1995) Immunization with the paraneoplastic encephalomyelitis antigen HuD does not cause neurologic disease in mice. Neurology, 45, 1873-1878.

Sodeyama, N. et al. (1999) Pattern of epitopic reactivity of the anti-Hu antibody on $\mathrm{HuD}$ with and without paraneoplastic syndrome. J Neurol Neurosurg Psychiatry, 66, 97-99.

Totland, C. et al. (2011) CDR2 antigen and Yo antibodies. Cancer Immunol Immunother, 60, 283-289. Winter, S.F. et al. (1993) Antibodies against autologous tumor cell proteins in patients with small-cell lung cancer: association with improved survival. J. Natl. Cancer Inst.J Natl Cancer Inst, 85, 2012-2018. Yamamoto, A. et al. (1998) Deficiency in protein L-isoaspartyl methyltransferase results in a fatal progressive epilepsy. Journal of Neuroscience, 18, 2063-2074.

Yang, M.L. et al. (2006) Intracellular protein modification associated with altered T cell functions in autoimmunity. Journal of Immunology, 177, 4541-4549.

Zhu, J.X. et al. (2006) Protein repair in the brain, proteomic analysis of endogenous substrates for protein L-isoaspartyl methyltransferase in mouse brain. Journal of Biological Chemistry, 281, 3380233813. 
Zirah, S. et al. (2006) Structural changes of region 1-16 of the Alzheimer disease amyloid beta-peptide upon zinc binding and in vitro aging. Journal of Biological Chemistry, 281, 2151-2161. 


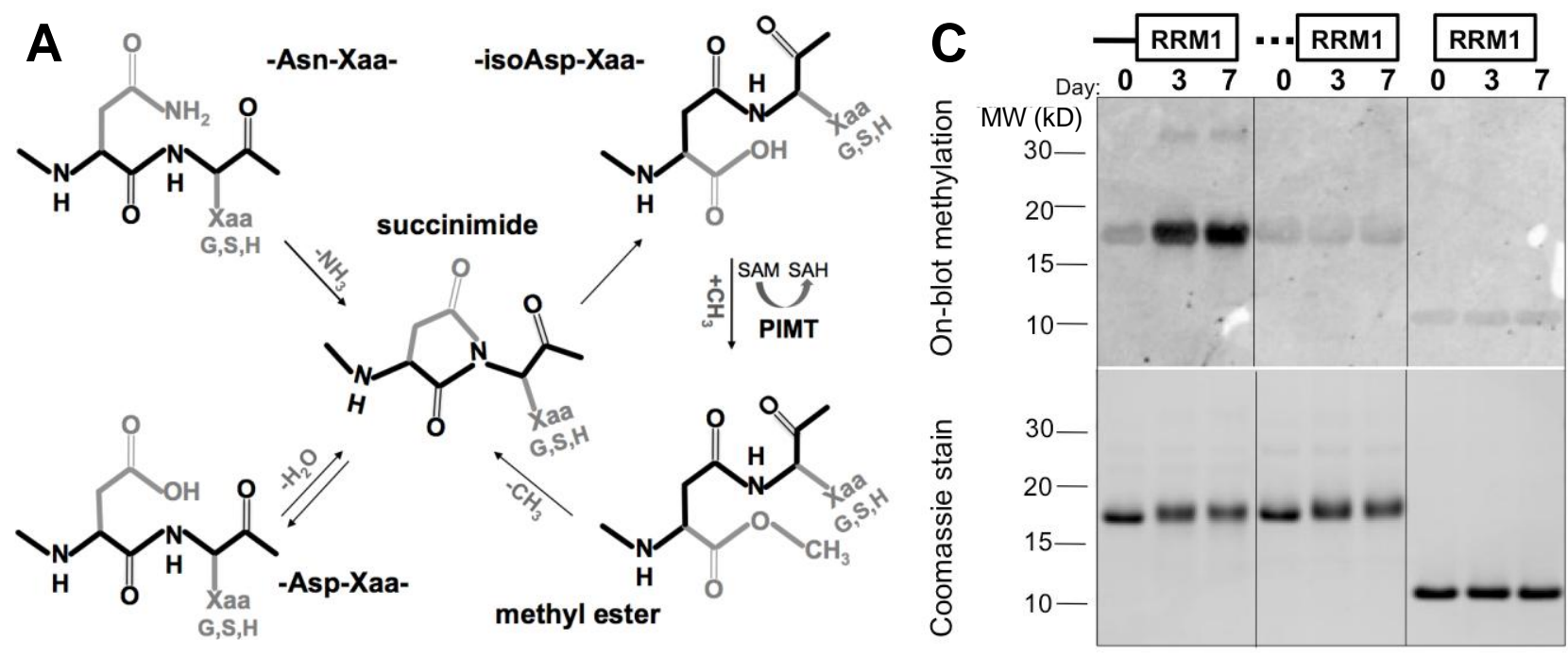

B

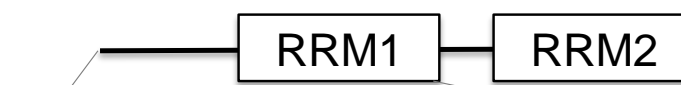

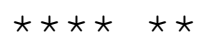

7

15

36

RRM3

MEPQVSNGPTSNTSNGPSSNNRNCPS PMQTGATTDDSKTNLIVNYLPQNMTQEEFRSLF GS I GE IESCKLVRDKITGQSLGYGFVNY I DPKDAEKA INTLNGLRLQTKTIKVSYARP

\section{Pulido et al. Figure 1}


A

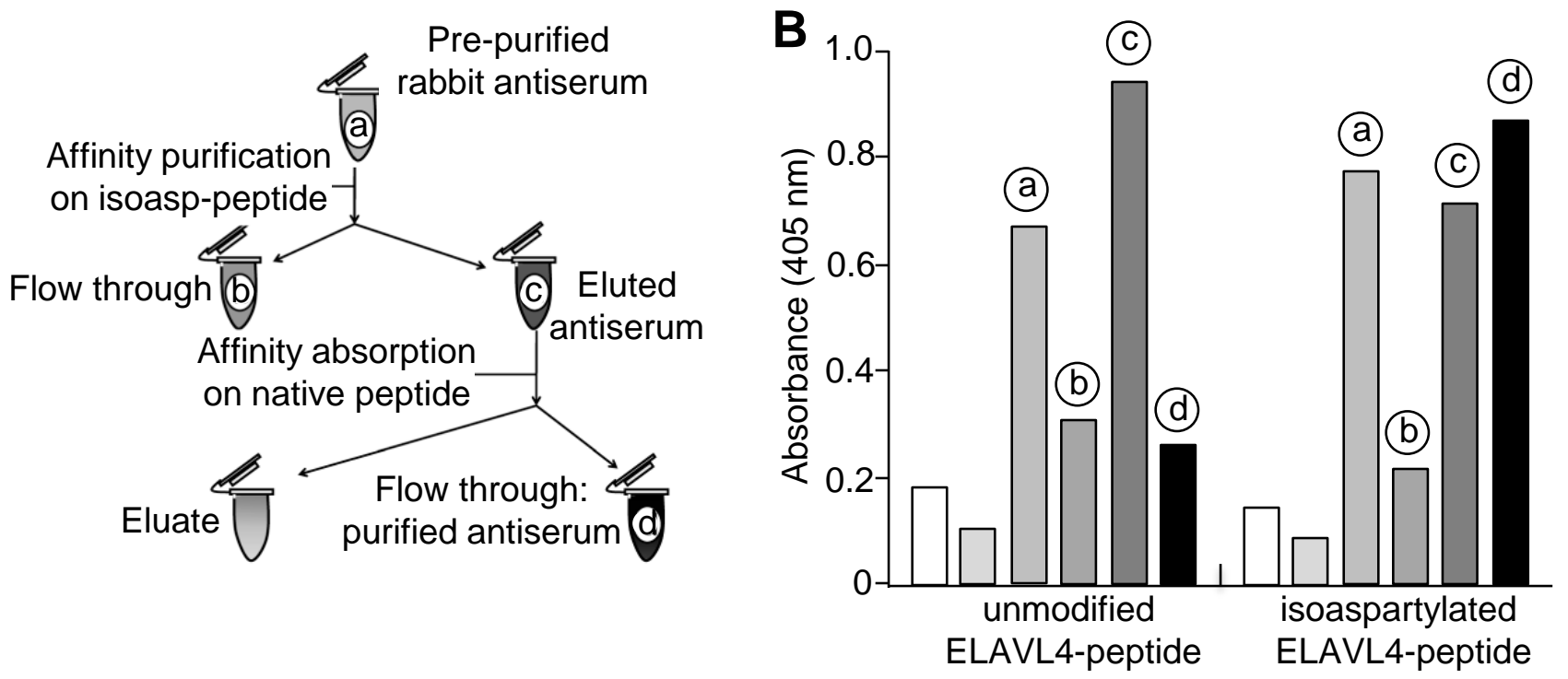

C $\begin{array}{lll}\text { wild type } & \mathbf{N}_{15} \mathbf{Q} & \mathbf{N}_{7} \mathbf{Q}+\mathbf{N}_{15} \mathbf{Q}\end{array}$

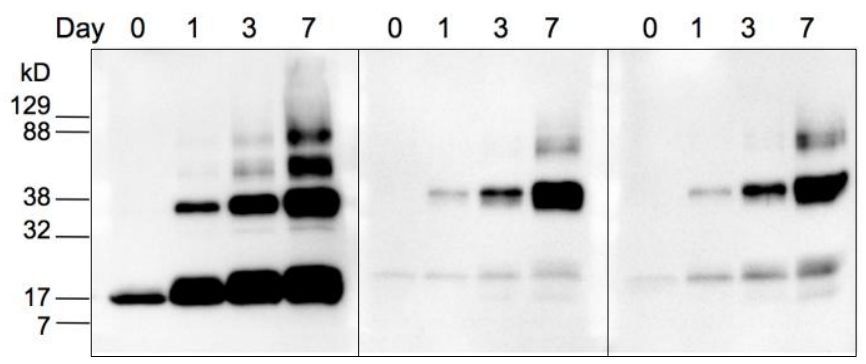

D

Competition 1000X ELAVL4 peptide

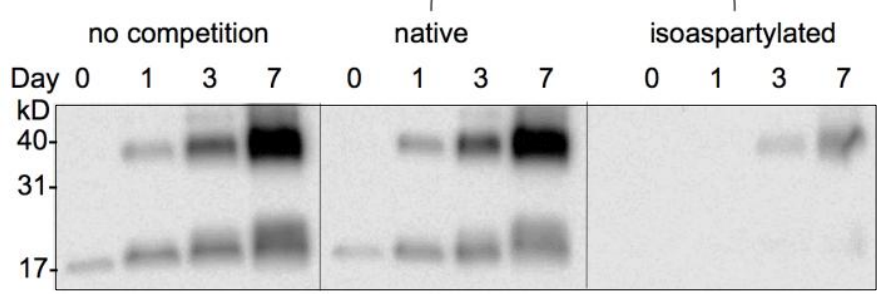


A

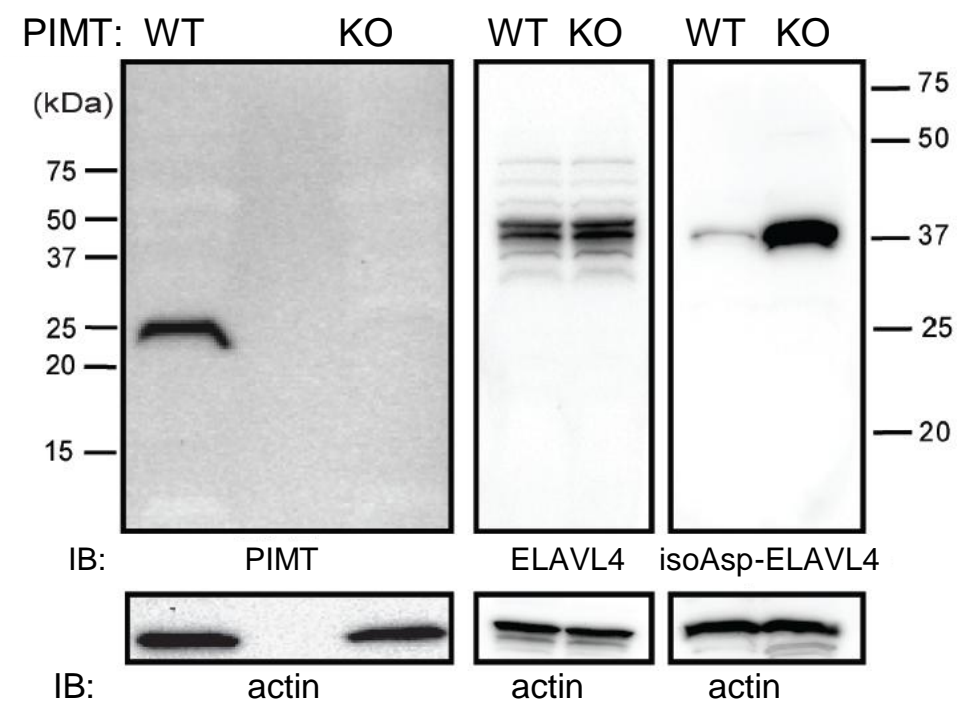

B Quantitation of anti-isoAsp-ELAVL4 reactivity

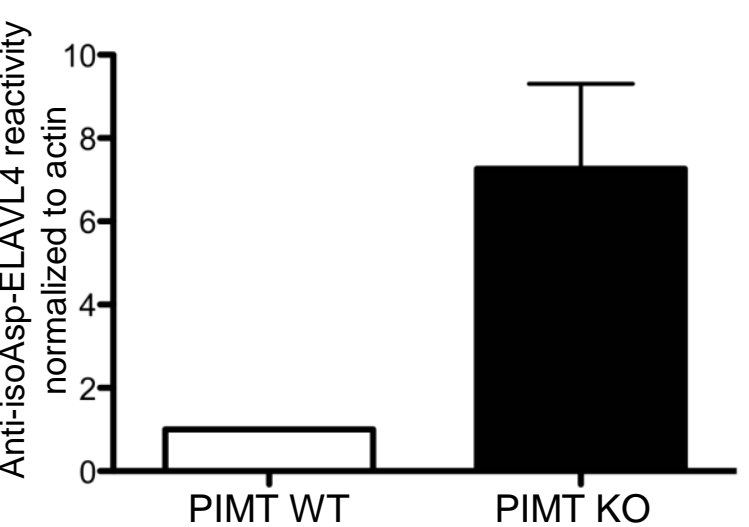

anti-isoAsp ELAVL4 with competition of $500 x$ molar excess

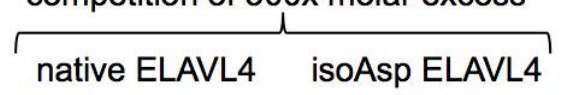

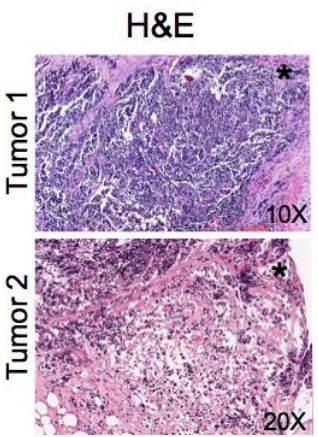

anti-ELAVL4 anti-isoAsp ELAVL4 anti-PIMT

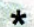

$20 X$
$10 x$

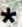

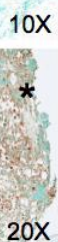

$10 \mathrm{x}$

*

$20 x$
$10 x$

0

10X

o

$20 x$

\section{Pulido et al. Figure 3}




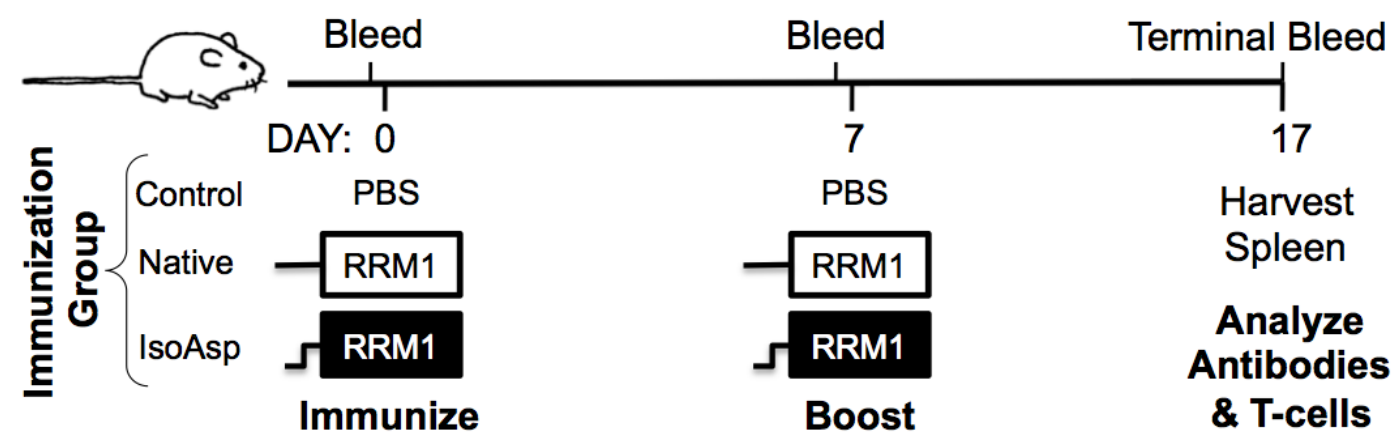

B

Immunization: PBS

pre- terminal immuniz. bleed

$(1: 2,000)(1: 40,000)$

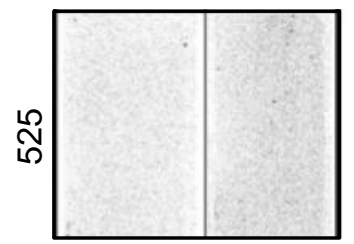

Nat Iso Nat Iso

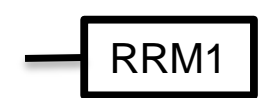

pre- pre- terminal immuniz. boost bleed $(1: 2,000) \quad(1: 2,000)(1: 40,000)$

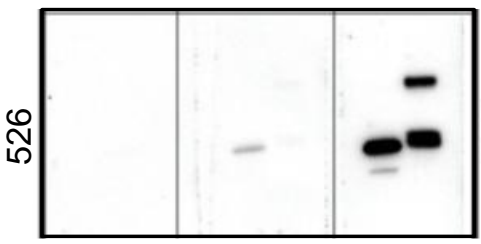

Nat Iso Nat Iso Nat Iso

\section{RRM1}

pre- pre- terminal immuniz. boost bleed $(1: 2,000)(1: 2,000)(1: 40,000)$

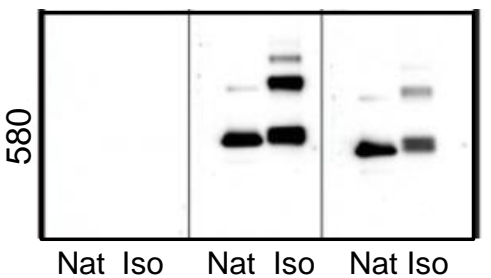

PBS

(reprobe)

anti-(His) $6 \mathrm{Tag}$

$(1: 20,000)$

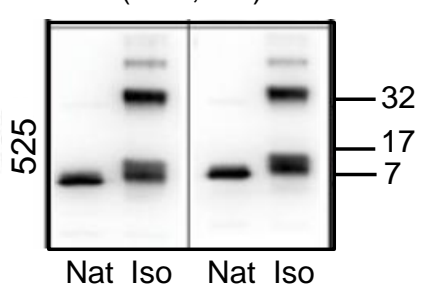

C

Mouse Antibody Response
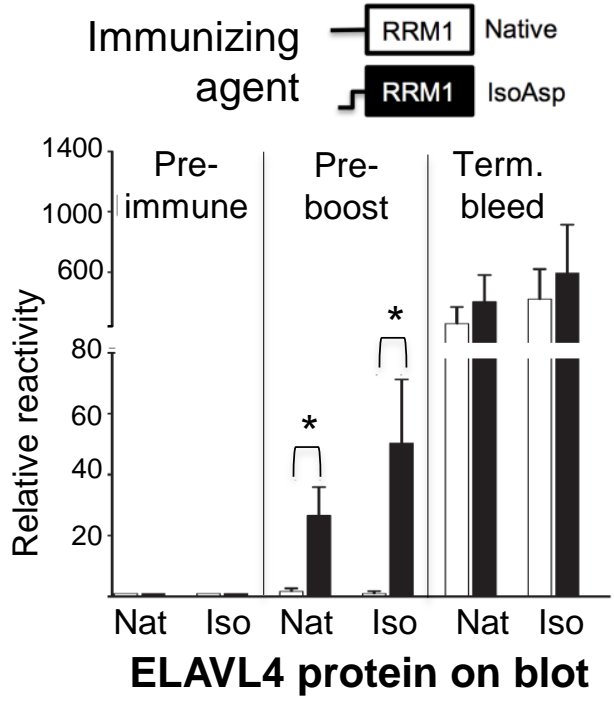

D

Mouse T-cell Response

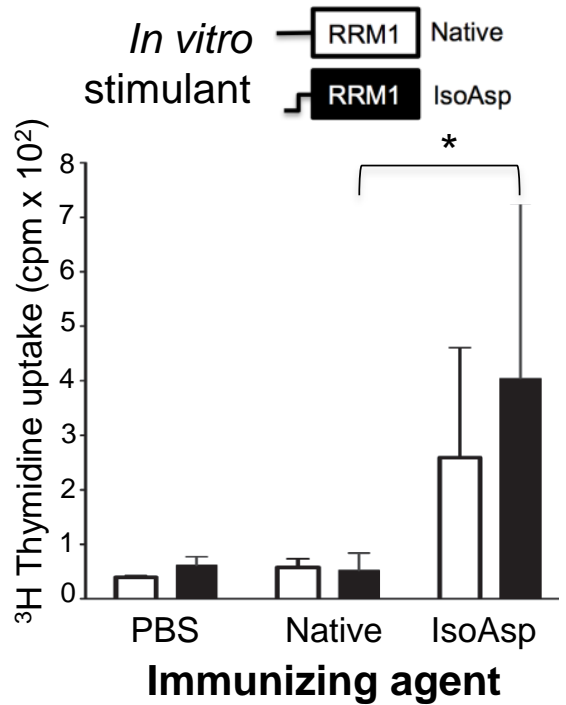

E

Human PBMC Response

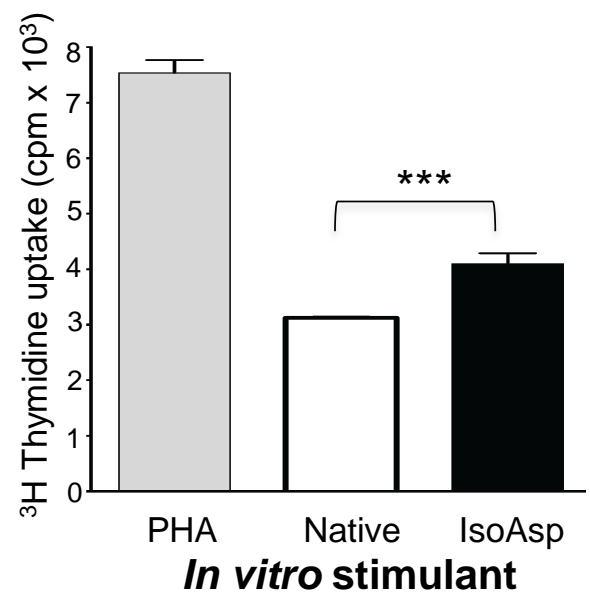



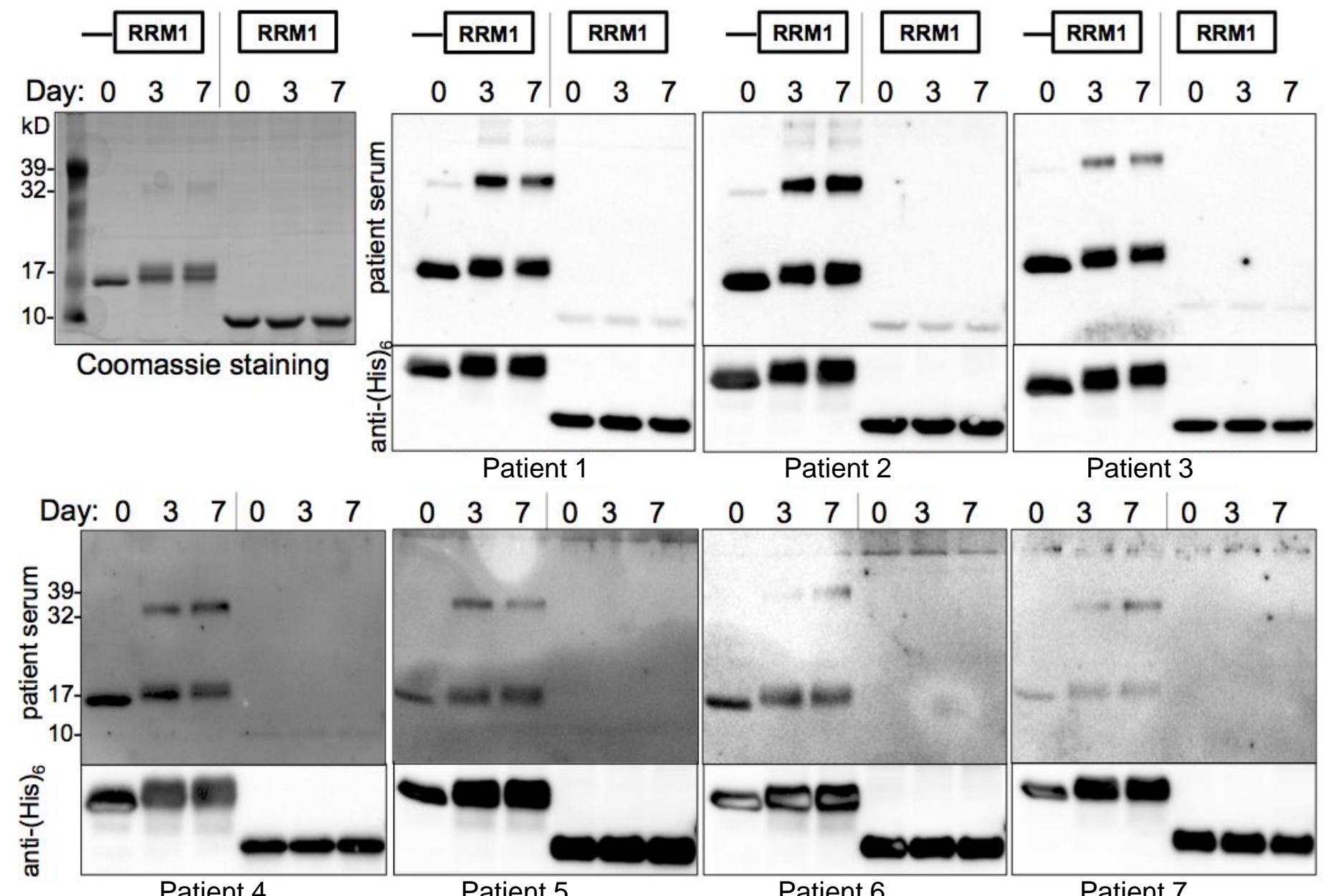

Patient 4

Patient 5

Patient 6

Patient 7

\section{Pulido et al. Figure 5}




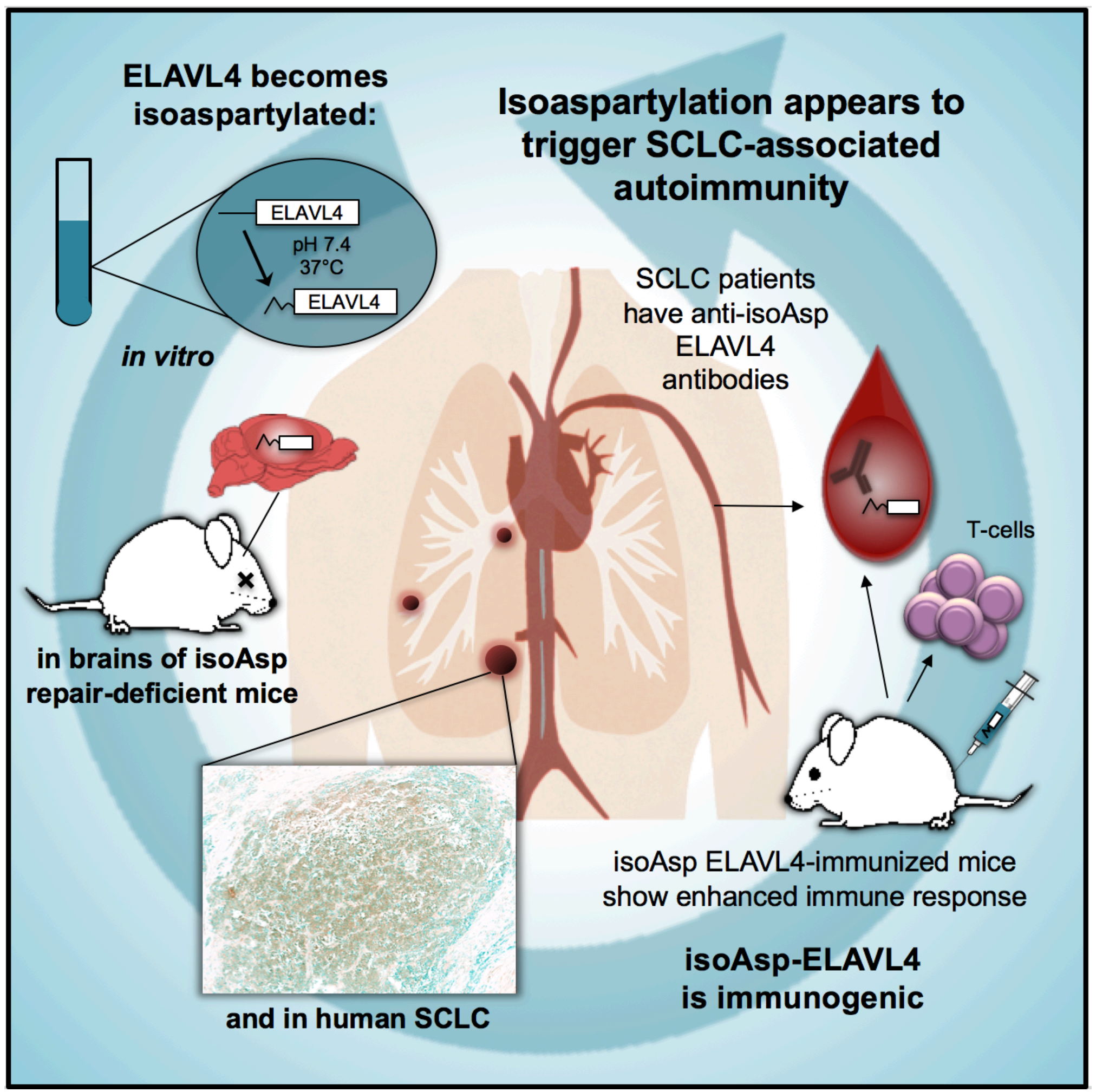

